\title{
Enhanced Measurement of Paper Basis Weight Using Phase Shift in Terahertz Time-Domain Spectroscopy
}

\author{
Mengbao Fan, ${ }^{1}$ Binghua Cao, ${ }^{2}$ and Guiyun Tian $^{3}$ \\ ${ }^{1}$ School of Mechatronic Engineering, China University of Mining and Technology, Xuzhou 221116, China \\ ${ }^{2}$ School of Information and Control Engineering, China University of Mining and Technology, Xuzhou 221116, China \\ ${ }^{3}$ School of Electrical and Electronic Engineering, Newcastle University, Newcastle upon Tyne NE1 7RU, UK \\ Correspondence should be addressed to Binghua Cao; caobinghua@cumt.edu.cn
}

Received 10 February 2017; Accepted 13 April 2017; Published 16 May 2017

Academic Editor: Dzung Dao

Copyright (C) 2017 Mengbao Fan et al. This is an open access article distributed under the Creative Commons Attribution License, which permits unrestricted use, distribution, and reproduction in any medium, provided the original work is properly cited.

\begin{abstract}
THz time-domain spectroscopy has evolved as a noncontact, safe, and efficient technique for paper characterization. Our previous work adopted peak amplitude and delay time as features to determine paper basis weight using terahertz time-domain spectroscopy. However, peak amplitude and delay time tend to suffer from noises, resulting in degradation of accuracy and robustness. This paper proposes a noise-robust phase-shift based method to enhance measurements of paper basis weight. Based on Fresnel Formulae, the physical relationship between phase shift and paper basis weight is formulated theoretically neglecting multiple reflections in the case of normal incidence. The established formulation indicates that phase shift correlates linearly with paper basis weight intrinsically. Subsequently, paper sheets were stacked to fabricate the samples with different basis weights, and experimental results verified the developed mathematical formulation. Moreover, a comparison was made between phase shift, peak amplitude, and delay time with respect to linearity, accuracy, and noise robustness. The results show that phase shift is superior to the others.
\end{abstract}

\section{Introduction}

Paper has been regarded as a potential material for paperbased sensors such as piezoresistive, thermoresistive, analytical, and clinical chemistry sensors due to softness, low cost, and lightweight, which opens a new venue of technologies [1-3]. Basis weight, the total weight per unit area of paper sheets, is one of the vital physical indexes for quality control of produced paper [4-6]. Currently, $\beta$-ray gauge is a broadly used technique in papermaking industries for basis weight measurement. It operates on the principle of transmitted or reflected $\beta$-ray attenuation. X-ray sensor is practically alternative for coating basis weight determination [7]. However, both $\beta$-ray and $\mathrm{X}$-ray are expensive due to the reduction of radiation for safety reasons, thus undesirable [6-8]. Aircoupled ultrasonic sensor [9] presents a noncontact and safe solution. Nonetheless, it requires powerful pulse excitations. In this context, noncontact, safe, and reliable measurement of paper basis weight is still an open problem.
Terahertz $(\mathrm{THz})$ frequency radiation lies between the millimeter and infrared regions of the electromagnetic spectrum. In contrast to $\beta$-ray and $\mathrm{X}$-ray radiations, $\mathrm{THz}$ radiation is nonionizing and therefore safe for humans $[10$, 11]. Also, $\mathrm{THz}$ waves could penetrate a wide variety of nonconducting materials that are opaque in the range of visible and infrared light [12]. It is noted that $\mathrm{THz}$ radiation share many similarities to ultrasonic in terms of measurement capabilities. However, THz radiation has shorter wavelength and does not need coupling media, thus enabling noncontact and online evaluation of thin films with better performances. $\mathrm{THz}$ time-domain spectroscopy (THz-TDS), which uses short-pulse $\mathrm{THz}$ radiation to probe material properties, has evolved as a promising spectroscopic technique for achieving high spectral resolution. With the aid of optical sampling, THz-TDS is capable deriving both the amplitude and phase signatures of $\mathrm{THz}$ transient electric field. Further, it is insensitive to background noises and produces a satisfactory signal with high SNR. Compared to the continuous-wave $\mathrm{THz}$ 
spectroscopy, THz-TDS provides rich information of samples due to the use of pulsed THz excitation. Therefore, THz-TDS has been considered as a quite promising tool to evaluate a part or plant nondestructively for quality control or structural integrity $[13,14]$.

Basically, determination of sample parameters can be regarded as an inverse electromagnetic problem and most inversion methods are classified as either iterative or noniterative [15-19]. Iterative approaches take parameter characterization as an optimization problem. They require accurate and efficient forward models like transfer function in the frequency domain and could infer multiple parameters simultaneously [17]. For instance, the refractive index and thickness of one paper sheet were obtained with time of flight, which is the difference between two adjacent peak position times of reflective signals [16]. Iterative approaches mainly differ in object functions and optimization methods which are tailored to specific applications [16-19]. An advantage of iterative approaches lies in simultaneous measurement of multiple parameters when the resultant changes of $\mathrm{THz}$ pulses are difficult to decouple. However, iterative approaches are subject to heavy computational burdens as they keep iteratively implementing forward models till they meet predefined convergence criteria [15]. In contrast, noniterative approaches, which need to seek for one or more appropriate features, are efficient and fairly popular. Noniterative approaches typically rely on the established relationship between the extracted signal features and the parameters of a sample, ignoring the underlying physical process [15]. Noniterative approaches are much more efficient than iterative approaches. However, noniterative approaches basically fail to decouple the $\mathrm{THz}$ pulses due to multiple parameters [16]. For THz-TDS, signal interpretations are done principally in the time and frequency domain. In the former case, peak amplitude and peak position time are the representative features [20-22]. Nonetheless, time-domain features like peak amplitude and peak position time are prone to be contaminated by noises, thus degrading accuracy. Fourier transform is a powerful tool to distinguish useful information and noises in the frequency domain [22, 23]. It is reported that phase imaging is more sensitive to the component pattern of a specimen than amplitude and timeof-flight imaging [24]. In [25], phase imaging is used to reveal the depth information of an optically opaque object and provides much better contrast for weak-absorption materials. The spectral phase, derived from a typical time-domain terahertz waveform by Fourier transform, is essentially linear [26]. For the case of a thin film, spectral phase is more sensitive than spectral amplitude [13]. Phase distribution allows the reconstruction of the Teflon object thickness pattern in low SNR registration conditions [27]. In summary, amplitude spectra are suitable for characterization of strong absorption materials [23], whereas phase spectra contain more significant information and exhibit linear behavior, thus outperforming amplitude spectra for weak-absorption and transparent materials, low SNR, and thin-film cases [24-26].

In our previous work, peak amplitude and delay time were employed to gauge paper basis weight in a least squares [28]. The results show that delay time is superior to peak

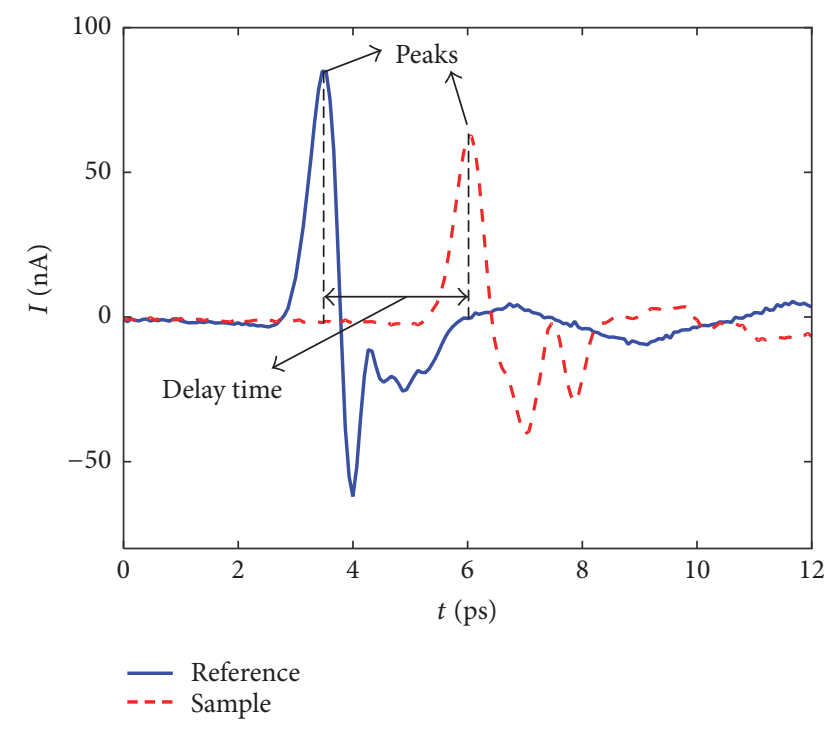

FIgURE 1: Typical THz-TDS signals.

amplitude. However, the adopted peak amplitude and delay time belong to time-domain features, thus prone to suffer from environmental noises. Moreover, amplitude spectra are unfeasible owing to low absorption of paper sheets in the $\mathrm{THz}$ region. We hereby propose to adopt phase shift from $\mathrm{THz}$-TDS pulses to measure paper basis weight for accuracy enhancement. The remainder of this work is arranged as follows. Section 2 mathematically formulates the relationship between phase shift and paper basis weight and basically introduces peak amplitude, delay time, and phase-shift based methods. Next, the experimental setup is shown and samples were fabricated in Section 3. In Section 4, experiments were performed to verify the linearity of the proposed phase-shift method, which matches well with the established formulation in Section 2. Then, correlation coefficients were calculated for variable selection. Subsequently, a comparison was made between phase shift, peak amplitude, and delay time features in terms of accuracy and noise robustness. Finally, conclusions are outlined in Section 5.

\section{Principle of Paper Basis Weight Measurement}

When a $\mathrm{THz}$ pulse propagates through a slab, it will be modulated due to the dispersion and absorption of the slab, as shown in Figure 1. It is observed that the slab decreases peak intensity of the $\mathrm{THz}$ pulse. Simultaneously, the peak position of the $\mathrm{THz}$ pulse is delayed as well. The amplitude and phase spectra of the $\mathrm{THz}$ pulse are readily obtained by using a FFT algorithm.

There is a need to develop the mathematical relationship between paper basis weight and modulated $\mathrm{THz}$ pulses. Basically, there are multiple propagation paths in a dielectric sample resulting from the refraction and reflection at the interfaces between the sample and free space. In the case of normal incidence, however, no reflections and scattering losses could be assumed in a $\mathrm{THz}$ pulse [29]. Based on 


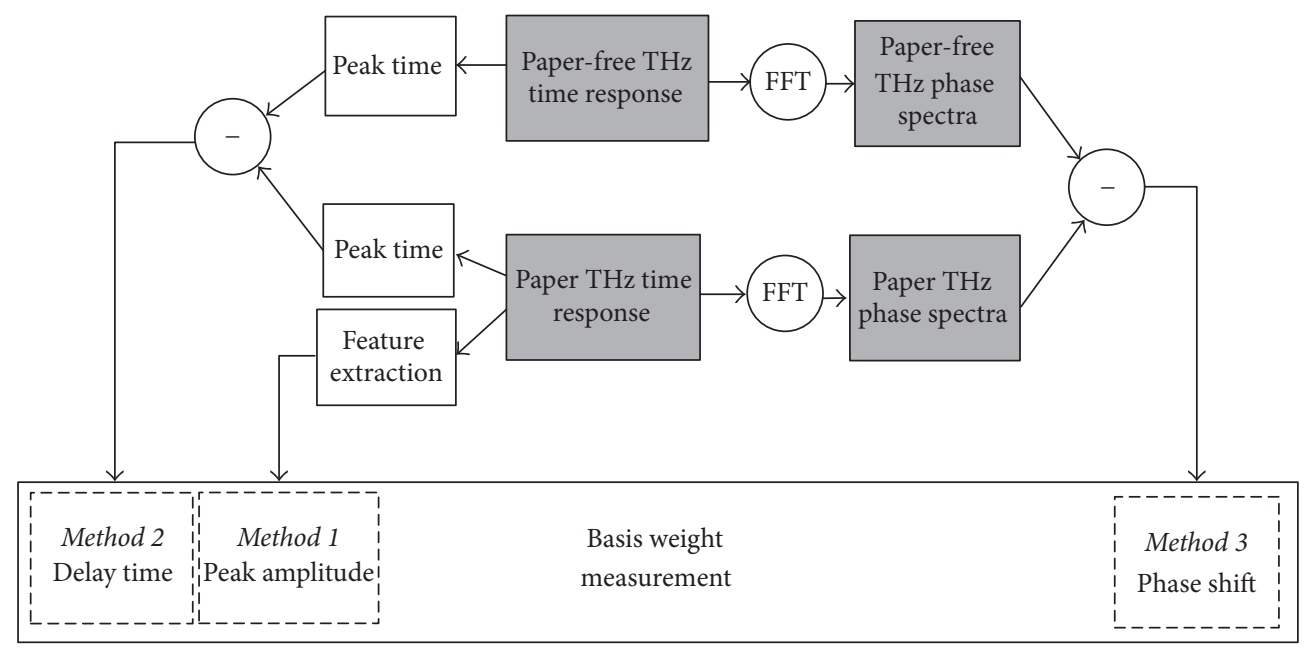

FIGURE 2: Flowchart of the presented three methods.

the Fresnel Formulae, the $\mathrm{THz}$ amplitude transmittance $T$ for most low-loss materials can be expressed as [30]

$$
\begin{aligned}
T(f) & =\frac{E_{\mathrm{sam}}(f)}{E_{\mathrm{ref}}(f)}=A \exp \left(-i \varphi_{d}\right) \\
& \approx \frac{4 n}{(1+n)^{2}} \exp \left[\frac{i 2 \pi f(\tilde{n}-1) d}{c}\right],
\end{aligned}
$$

where $E_{\text {ref }}(f)$ and $E_{\text {sam }}(f)$ are the incident and transmitted $\mathrm{THz}$ amplitude spectra, respectively; $\varphi_{d}$ is the phase shift between the reference and sample THz pulses; $\widetilde{n}=n+i \kappa$ is the complex refractive index of the sample; $d$ is the sample thickness; $f$ is the frequency; and $c$ is the speed of light in vacuum.

In this work, $E_{\text {ref }}(f)$ and $E_{\text {sam }}(f)$ are the THz amplitude of the reference and sample, respectively. Thus the refractive index can be obtained using (1):

$$
n(f)=\frac{\varphi_{d}(f) c}{2 \pi f d}+1
$$

Using (2), we can deduce the relationship between the phase shift $\varphi_{d}(f)$ and sample thickness $d$

$$
\varphi_{d}(f)=\frac{2 \pi f[n(f)-1]}{c} \cdot d .
$$

Equation (3) implies that the phase shift $\varphi_{d}(f)$ is proportional to the sample thickness $d$ for a certain frequency. The refractive index at the frequency $f$ is supposed to be constant.

Give that the mass density and thickness of paper sheets are $\rho$ and $d$, respectively. By definition, basis weight BW is the weight per unit area of paper sheets.

$$
\mathrm{BW}=\rho \cdot d .
$$

Substituting (4) into (3) yields

$$
\varphi_{d}(f)=\frac{2 \pi f[n(f)-1]}{c \rho} \cdot \mathrm{BW} .
$$

As shown in Figure 2, three methods using phase shift, peak amplitude, and delay time features are presented. Paperfree $\mathrm{THz}$ time response is acquired as the reference at the absence of paper sheets, while paper $\mathrm{THz}$ time response is derived for prediction of paper basis weight. First, peak amplitude is extracted from paper $\mathrm{THz}$ time response in method one. In method two, delay time is computed by subtracting peak time of paper-free $\mathrm{THz}$ time response from that of paper $\mathrm{THz}$ time response. Next, paper-free $\mathrm{THz}$ time response is changed to paper-free $\mathrm{THz}$ frequency response, and paper $\mathrm{THz}$ time response is changed to paper $\mathrm{THz}$ frequency response by FFT transform, which generates $\mathrm{THz}$ phase spectra accordingly. Afterwards, the phase shift due to paper sheets is computed as the feature in method three by subtracting paper phase spectra from paper-free phase spectra. Linear least squares fitting technique will be employed to fit a model which relates paper basis weight of the samples and phase-shift features from the experiments. All the presented methods will be applied to infer paper basis weight in the following sections and conclusions will be drawn finally.

\section{Experimental Setup and Samples}

Figure 3 shows the schematic diagram of a THz-TDS system in transmission configuration. The THz-TDS setup Z1 (Zomega Terahertz Corporation, New York, USA) mainly consists of a mode-locked Ti: sapphire (ML Ti:S) laser oscillator, a large-aperture GaAs photoconductive antenna (PCA) for $\mathrm{THz}$ generation, and Zinc Telluride (ZnTe) for $\mathrm{THz}$ detection. The laser has a repetition rate of $80 \mathrm{MHz}$ and duration of $68 \mathrm{fs}$ pulse at $800 \mathrm{~nm}$. It is split into a pump beam and a probe beam with a cubic beam splitter. The pump beam illuminates the large-aperture GaAs PCA to produce roughly single-cycle $\mathrm{THz}$ pulses with a duration on the order of one picosecond, so the frequency of the produced $\mathrm{THz}$ pulses falls in the $\mathrm{THz}$ range. The emitted $\mathrm{THz}$ pulses then propagate through a series of off-axis parabolic mirrors 
TABLE 1: Sample parameters.

\begin{tabular}{lccccccc}
\hline \multirow{2}{*}{ Parameters } & \multicolumn{9}{c}{ Sample number } \\
& 1 & 2 & 3 & 4 & 5 & 6 & 7 \\
\hline Office paper $80 \mathrm{~g} / \mathrm{m}^{2}$ & & & & & & \\
Basis weight $\left(\mathrm{g} / \mathrm{m}^{2}\right)$ & 80 & 160 & 240 & 320 & 400 & 480 & 560 \\
$\quad$ Thickness $(\mathrm{mm})$ & 0.108 & 0.216 & 0.324 & 0.432 & 0.540 & 0.648 & 0.756 \\
Office paper $160 \mathrm{~g} / \mathrm{m}^{2}$ & & & & & & \\
Basis weight $\left(\mathrm{g} / \mathrm{m}^{2}\right)$ & 160 & 320 & 480 & 640 & 800 & 960 & 1120 \\
Thickness $(\mathrm{mm})$ & 0.168 & 0.336 & 0.504 & 0.672 & 0.840 & 1.008 & 1.176 \\
\hline
\end{tabular}

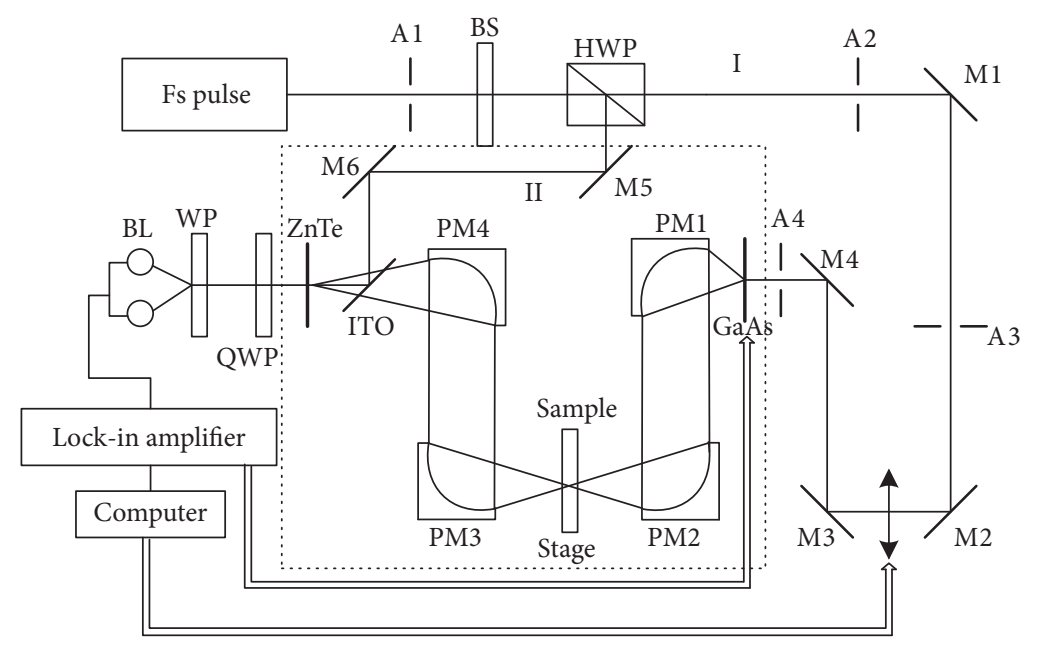

FIGURE 3: Schematic diagram of the THz-TDS experimental system. BS: beam splitter, PM: parabolic mirror, HWP: half-wave plate, QWP: quarter-wave plate, WP: Wollaston prism, BL: balanced photodiode, A: aperture, and M: mirror.

and are focused onto a Zinc Telluride (ZnTe) detector. The $\mathrm{THz}$ beam modifies the index ellipsoid of the ZnTe crystal transiently via the Pockels Effect. The linearly polarized probe beam copropagates inside the crystal with $\mathrm{THz}$ beam, and its phase is modulated by the refractive index change induced by the electric field of $\mathrm{THz}$ pulses. This phase change is converted to an intensity change by a Wollaston prism. In the experimental setup, a pair of balanced detectors in differential mode is used to suppress the common mode laser noises. As a consequence, the balanced detectors basically remain in equilibrium till the samples under test break it. The output current of the balanced detectors is directly proportional to $\mathrm{THz}$ electric field due to the samples. A mechanical delay line is used to change the delay time between a $\mathrm{THz}$ pulse and probe pulse, and THz electric-field waveform can be obtained by scanning this delay time and performing a repetitive sampling measurement. To increase the sensitivity, the pump beam is modulated by a mechanical chopper, and the $\mathrm{THz}$ field-induced modulation of the probe beam is extracted by a lock-in amplifier.

The samples were manufactured with two kinds of normal printer paper sheets. One has $80 \mathrm{~g} / \mathrm{m}^{2}$ basis weight and $0.108 \mathrm{~mm}$ thickness and the other has $160 \mathrm{~g} / \mathrm{m}^{2}$ basis weight and $0.168 \mathrm{~mm}$ thickness. Basically, air gaps between the stacked paper sheets would change $\mathrm{THz}$ pulses due to multiple reflections and transmissions. To reduce the influences of air gaps, we pressed completely the stacked paper sheets and fixed them with four thumbtacks on a hard paperboard which has a $13 \mathrm{~mm}$ hole in diameter. In such a way, the fabricated samples were made as homogeneous as possible. Hence, air gaps should impose negligible impact on $\mathrm{THz}$ pulses.

Then, the fabricated samples with different basis weights are listed in Table 1. To know the thickness of one paper sheet, we gauged 30 stacked paper sheets with a digital micrometer caliper. Then, the thickness of one paper sheet was determined by dividing the reading by 30 . Before the inspections, the moisture was evaporated from the fabricated samples to eliminate the influence of inhomogeneous water distribution. The surrounding temperature and relative humidity were $25^{\circ} \mathrm{C}$ and $40 \%$, respectively. No provision is made for filling the box with dry nitrogen. In our experiments, each recorded $\mathrm{THz}$ pulse contains 201 data points.

\section{Results and Discussion}

4.1. THz Pulses of Samples. The experiments were carried out and the THz pulses due to the $80 \mathrm{~g} / \mathrm{m}^{2}$ and $160 \mathrm{~g} / \mathrm{m}^{2}$ samples were acquired, respectively. Figure 4 illustrates the $\mathrm{THz}$ pulses from the stacked paper sheets. The curves labeled ref are the $\mathrm{THz}$ pulses of the reference ( $\mathrm{THz}$ wave transmitted through the air $)$ and those labeled $k(k=1,2, \ldots, 8)$ are the THz pulses 


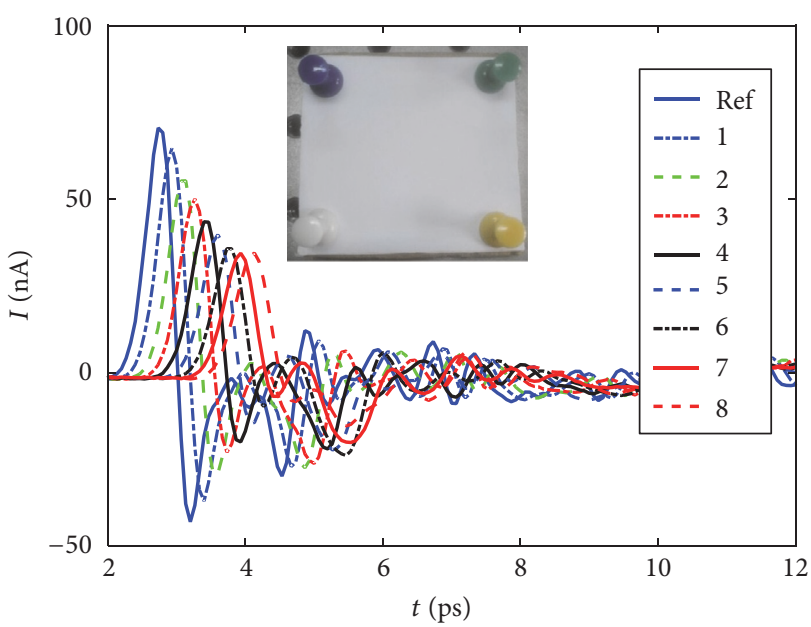

(a) $80 \mathrm{~g} / \mathrm{m}^{2}$

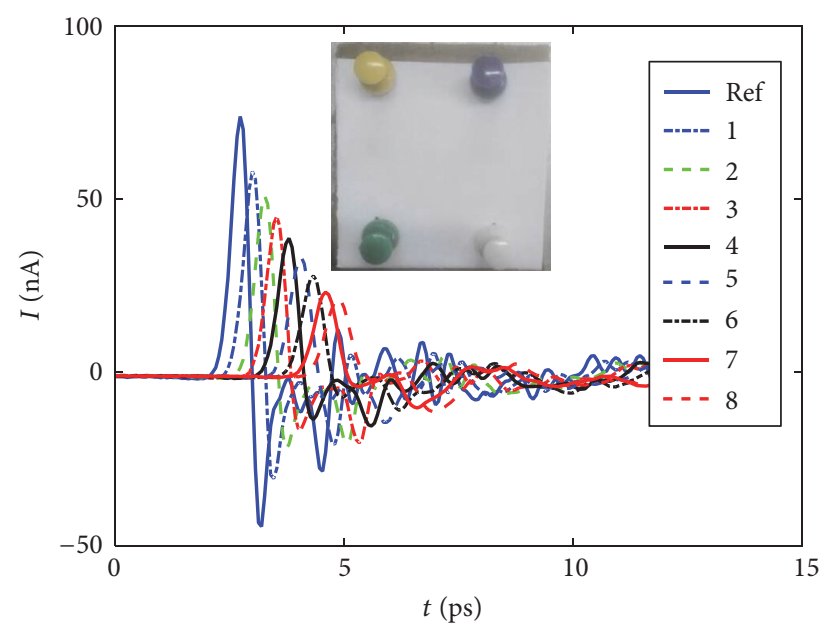

(b) $160 \mathrm{~g} / \mathrm{m}^{2}$

FIGURE 4: THz pulses of samples.

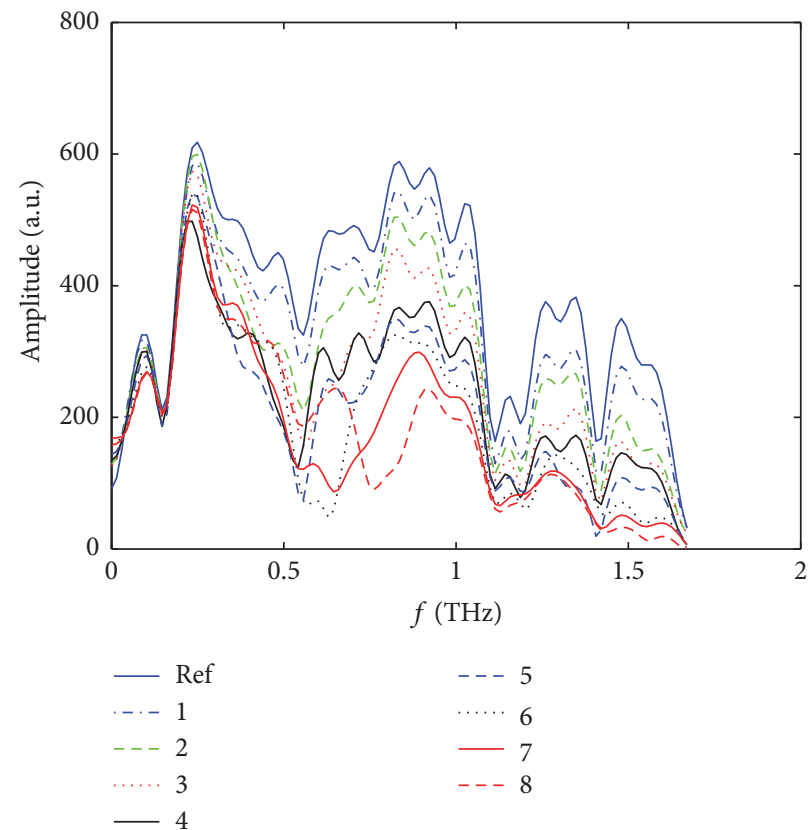

(a) Amplitude spectra

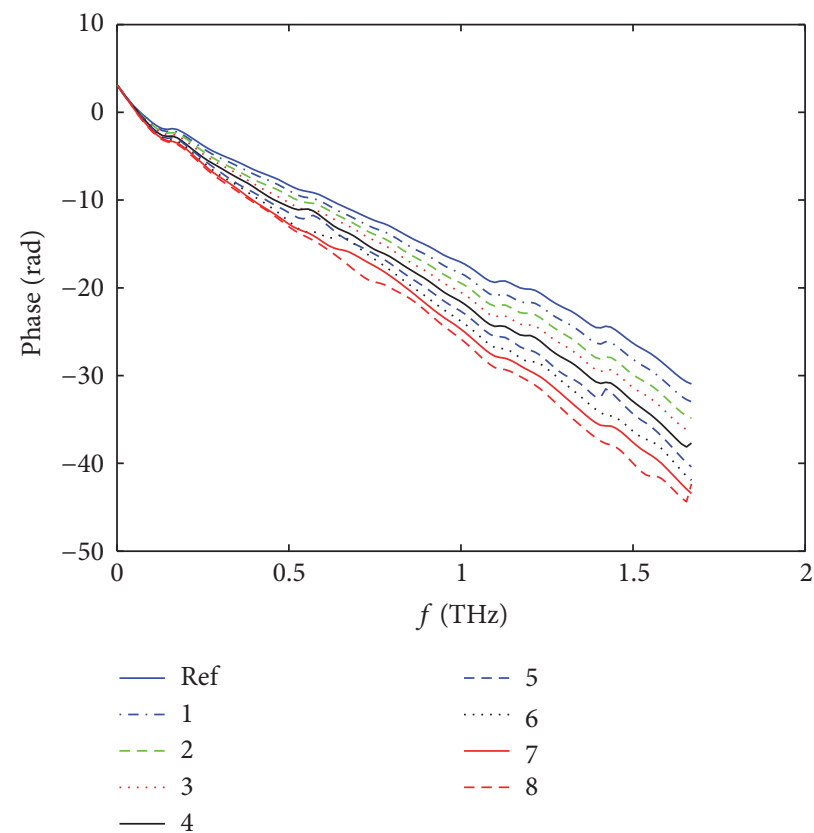

(b) Phase spectra

FIGURE 5: THz spectral responses of $80 \mathrm{~g} / \mathrm{m}^{2}$ samples.

transmitted through the samples $k$ which consist of $k$ pieces of paper sheets.

Along with the increase of paper basis weight, the acquired $\mathrm{THz}$ pulses are attenuated in amplitude and delayed in time. Peak amplitude and delay time correspond to the height and position of the peaks of the $\mathrm{THz}$ pulses. They are changeable owing to random noises. After Fourier analysis, the amplitude and phase spectra of the $\mathrm{THz}$ pulses were obtained, as illustrated in Figures 5 and 6, respectively. It is observed that both amplitude and phase spectra change with paper basis weight. Phase spectra vary monotonically, whereas amplitude spectra do not. This finding implies that phase spectra are supposed to outperform amplitude spectra for measurement of paper basis weight.

According to the proposed phase-shift based method in Section 2, phase shift $\varphi_{d}(f)$ is obtained by

$$
\varphi_{d}(f)=\varphi_{\text {ref }}(f)-\varphi_{\text {paper }}(f),
$$

where $\varphi_{\text {ref }}(f)$ and $\varphi_{\text {paper }}(f)$ are the phase spectra of the $\mathrm{THz}$ pulses with and without samples, respectively.

Figure 7 shows the spectra of phase shift due to the samples. As one can see, phase shift increases with the increase of basis weight of the samples. Basically, phase shift of each 


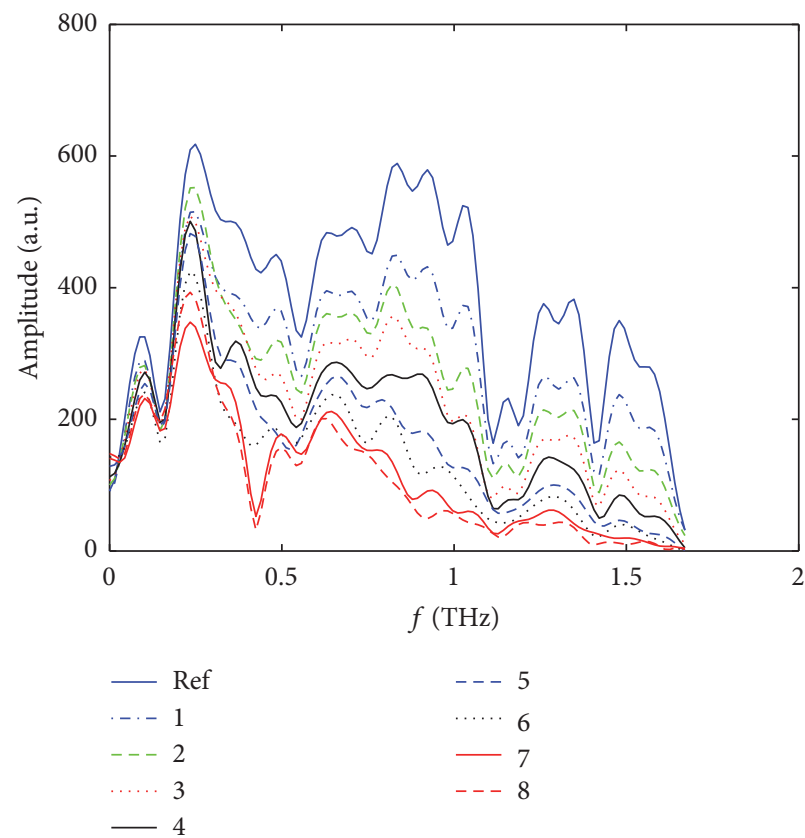

(a) Amplitude spectra

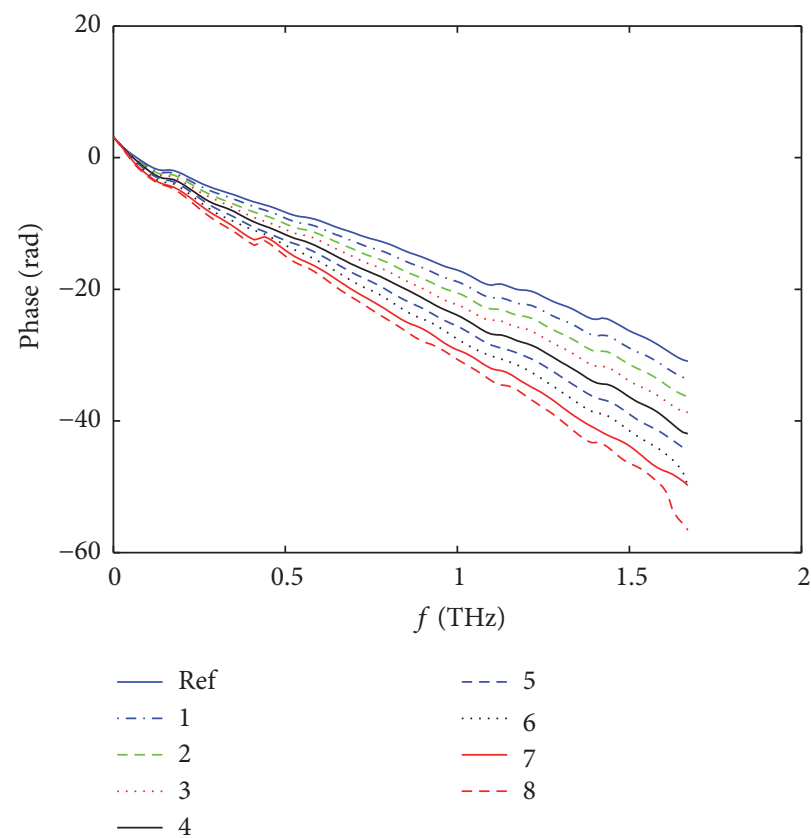

(b) Phase spectra

Figure 6: THz spectral responses of $160 \mathrm{~g} / \mathrm{m}^{2}$ samples.

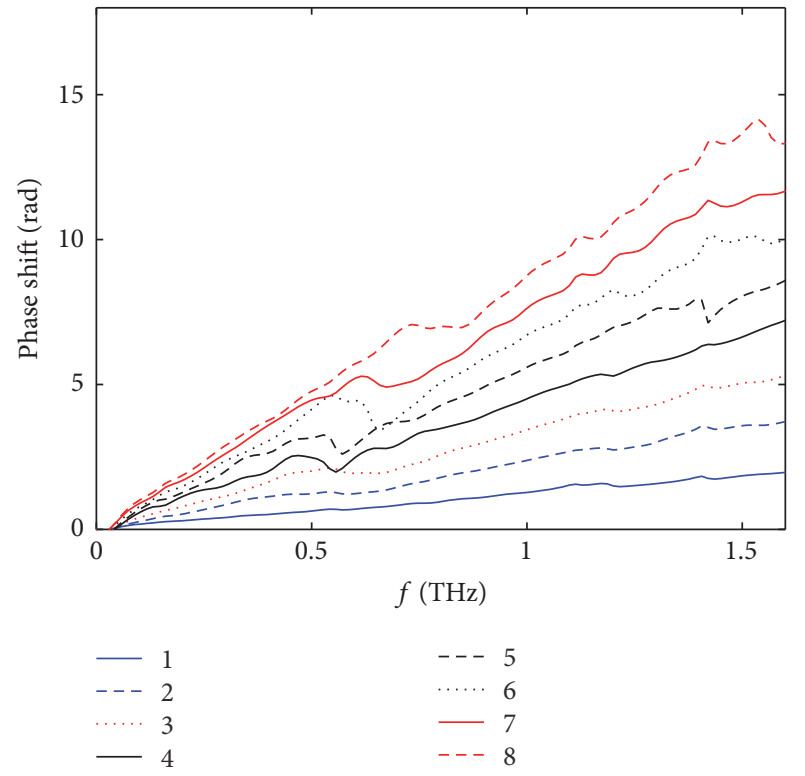

(a) $80 \mathrm{~g} / \mathrm{m}^{2}$
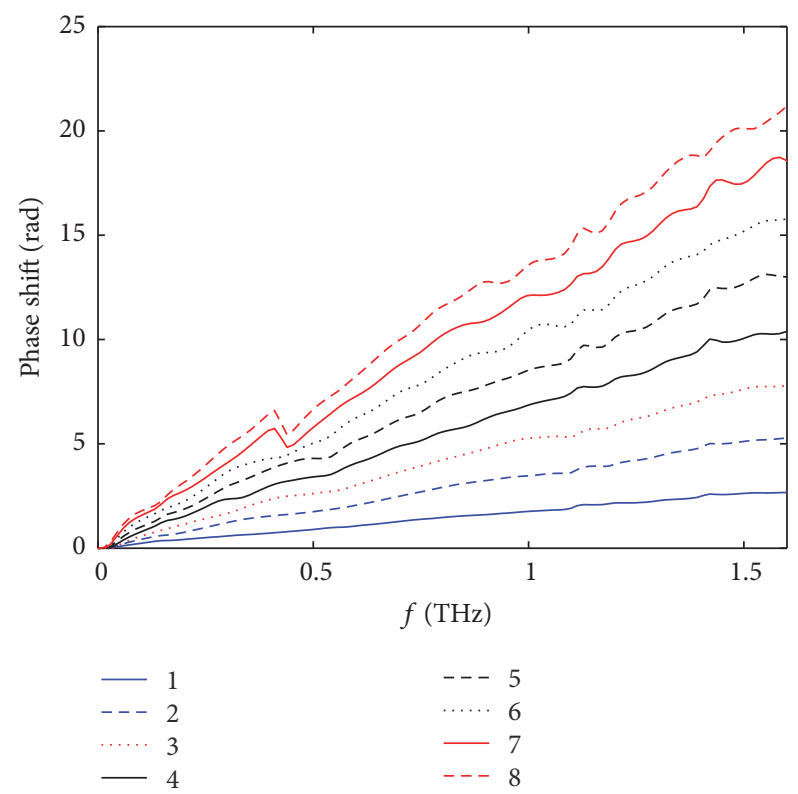

(b) $160 \mathrm{~g} / \mathrm{m}^{2}$

FIGURE 7: Phase shift of samples.

frequency component could be used for determination of paper basis weight.

From (5), the measurement sensitivity is

$$
\frac{\partial \varphi_{d}(f)}{\partial \mathrm{BW}}=\frac{2 \pi f[n(f)-1]}{c \rho} .
$$

Equation (7) shows that the higher the frequency of phase shift, the higher the sensitivity.
4.2. Linearity Evaluation for Variable Selection. There are hundreds of frequency components available in a derived spectral $\mathrm{THz}$ pulse. Nonetheless, the raw THz spectral signals contain both high signal-to-noise ratio (SNR) and low SNR information, along with various types of noises. Therefore, some components are not suitable to gauge paper basis weight. The determination of a proper frequency component is typically called variable or wavelength selection [31], which holds great importance for quantitative accuracy. Variable 


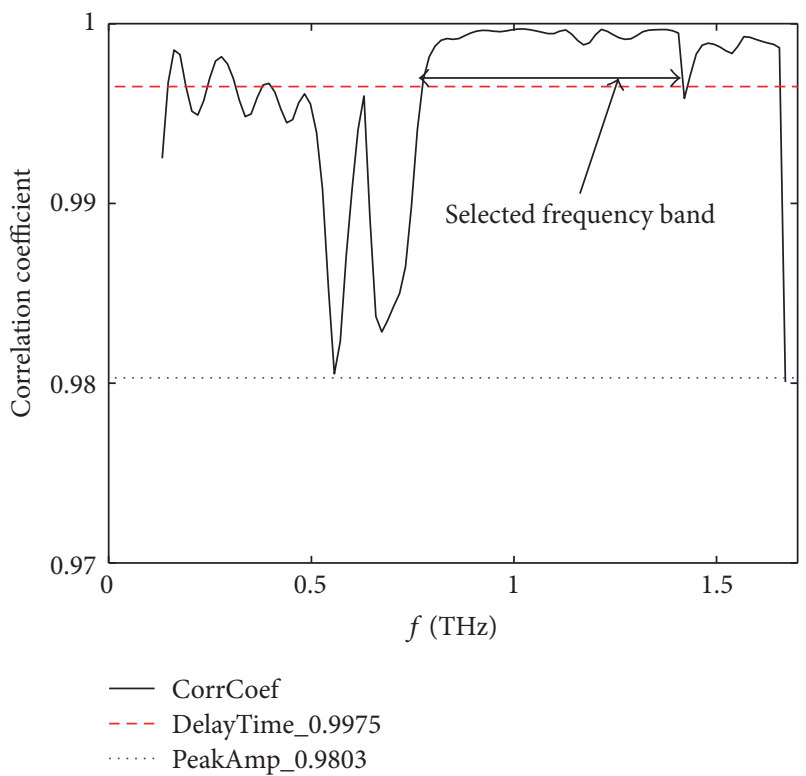

(a) $80 \mathrm{~g} / \mathrm{m}^{2}$ samples

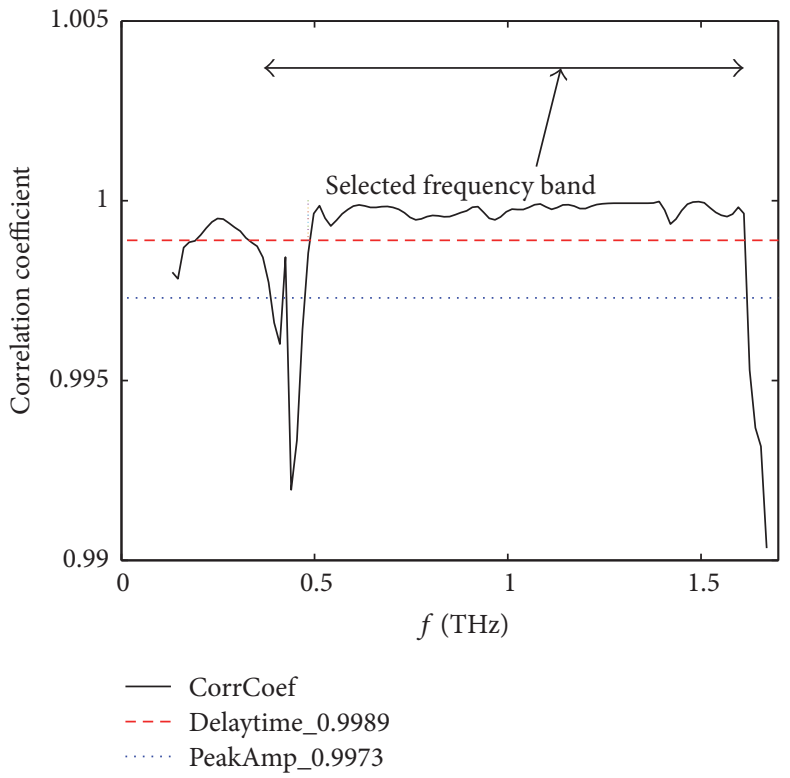

(b) $160 \mathrm{~g} / \mathrm{m}^{2}$ samples

FIGURE 8: Correlation coefficients for evaluation of components.

selection can remove the influences of inapplicable frequency components. In this way, a proper frequency component would be determined, and its phase shift would be utilized to predict the basis weight of paper sheets.

Correlation coefficient is a factor that measures the linear relationship between two variables. To evaluate the spectral features, correlation coefficient at each frequency was calculated using a single-term linear regression, as shown in Figure 8. For comparison, the correlation coefficients with peak amplitude and delay time were also computed and labeled in Figure 8.

From Figure 8, it is observed that only a few frequencies from the $\mathrm{THz}$ spectra have correlation coefficients smaller than 0.99 , which indicates that the majority of the frequency components in a spectral $\mathrm{THz}$ pulse are applicable to the measurement of paper basis weight due to intrinsic linearity. This observation matches well with the analysis in Section 2. The exceptional frequency components mainly fall in the range between $0.527 \mathrm{THz} 0.747 \mathrm{THz}$ for $80 \mathrm{~g} / \mathrm{m}^{2}$ samples and $0.408 \mathrm{THz} 0.575 \mathrm{THz}$ for $160 \mathrm{~g} / \mathrm{m}^{2}$ samples. These frequency components have poor informational quality, and they are not suitable to gauge paper basis weight measurement. The possible reason for this observation may come from the absorption of water vapor and unknown chemical substances in samples, since water vapor is reported to have two absorption peaks at around $0.557 \mathrm{THz}$ and $0.752 \mathrm{THz}$ [32]. The difference between exceptional frequency components from $80 \mathrm{~g} / \mathrm{m}^{2}$ and $160 \mathrm{~g} / \mathrm{m}^{2}$ samples may be mainly attributed to the difference between the chemical substances, to the authors' knowledge.

Figures 9 and 10 show the relationship between paper basis weight and peak amplitude, delay time, and phase shift, respectively. It is found that all the derived features follow a unique curve, which suggests that peak amplitude, delay time, and phase shift are practicable for measurement of paper basis weight.

Larger correlation coefficients will produce better prediction. Consequently, we selected the frequency components in $\mathrm{THz}$ spectra which have larger correlation coefficients than those of peak amplitude and delay time. The selected frequency bands are illustrated in Figure 8. To obtain the best prediction, the frequencies with the largest correlation coefficients are identified. The desirable frequencies are $1.011 \mathrm{THz}$ for $80 \mathrm{~g} / \mathrm{m}^{2}$ samples and $1.392 \mathrm{THz}$ for $160 \mathrm{~g} / \mathrm{m}^{2}$ samples, as shown in Figures 9 and 10, respectively.

For $80 \mathrm{~g} / \mathrm{m}^{2}$ samples, the correlation coefficients of peak amplitude and delay time are 0.9803 and 0.9975 , while the correlation coefficient at the selected frequency $1.011 \mathrm{THz}$ is 0.9997. For $160 \mathrm{~g} / \mathrm{m}^{2}$ samples, the correlation coefficients of peak amplitude and delay time are 0.9973 and 0.9989 , while the correlation coefficient at the selected frequency $1.392 \mathrm{THz}$ is 1.0. The comparison between the derived correlation coefficients shows that phase shift surpasses peak amplitude and delay time in terms of linearity. Therefore, phase-shift based method fundamentally allows more accurate measurement.

4.3. Accuracy Evaluation. In the following subsections, we intend to make a comparison between the three presented methods in terms of accuracy and noise robustness. For comparison, relative error $e_{f}$ is defined as

$$
e_{f}=\left|\frac{\mathrm{BW}_{\mathrm{pre}}^{f}-\mathrm{BW}_{\text {nom }}}{\mathrm{BW}_{\text {nom }}}\right| \times 100 \% \text {, }
$$

where $\mathrm{BW}_{\text {pre }}^{f}$ and $\mathrm{BW}_{\text {nom }}$ are the predicted and nominal basis weights, respectively. 


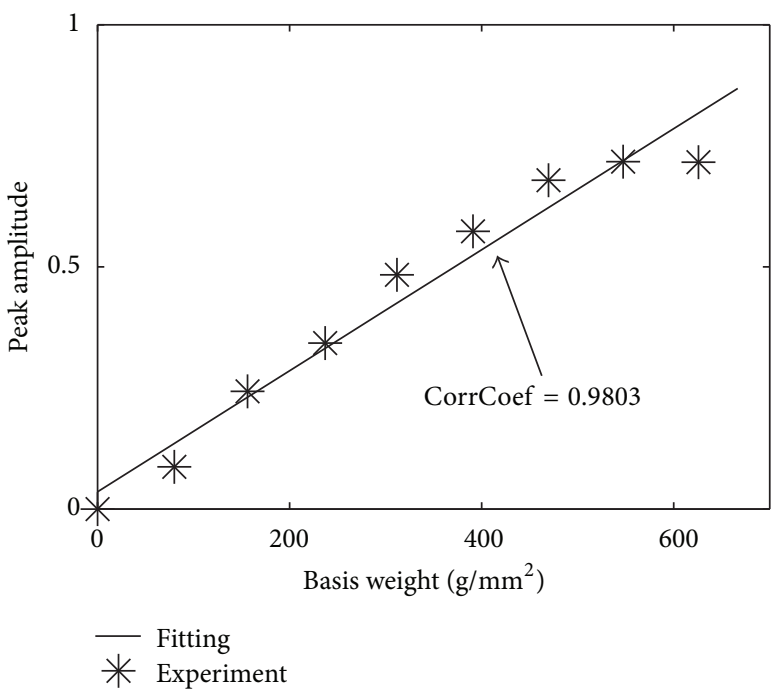

(a) Peak amplitude

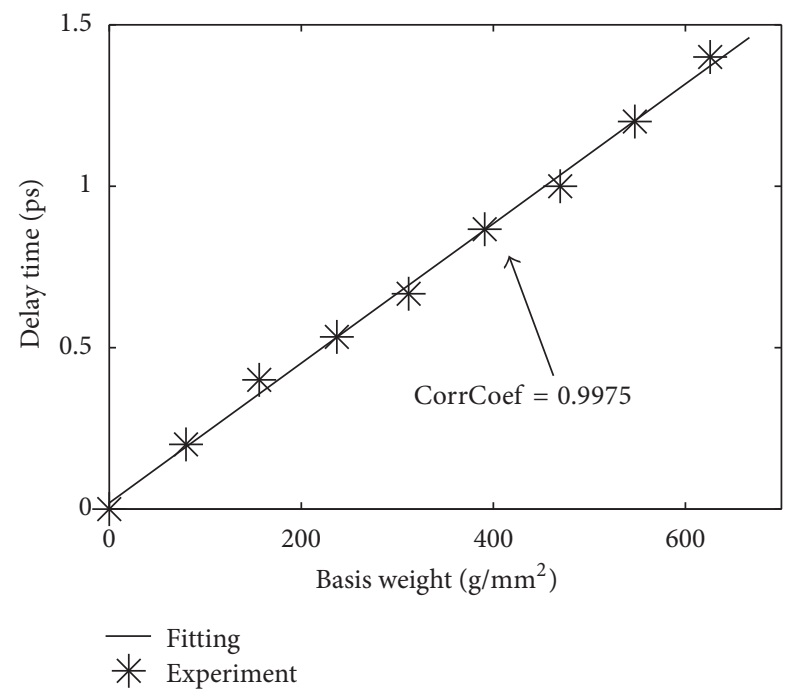

(b) Delay time

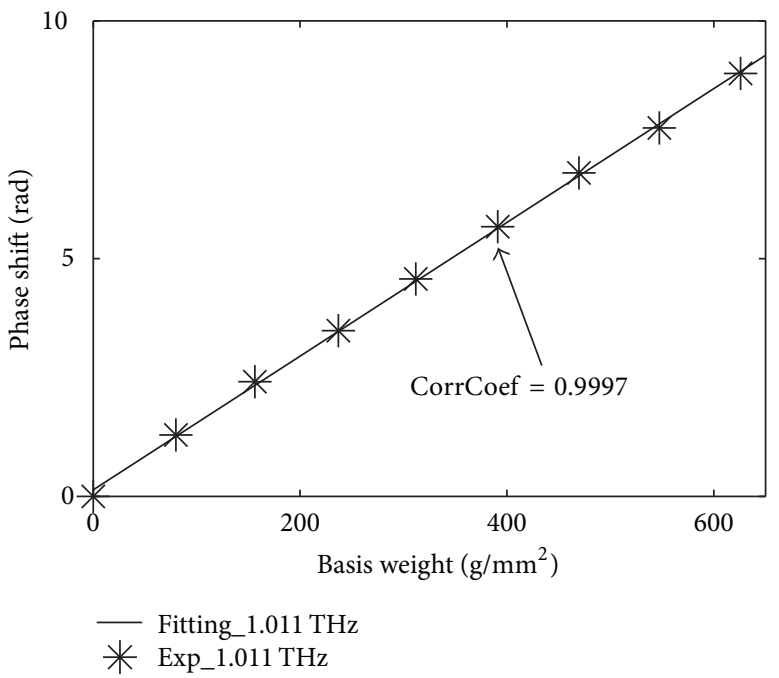

(c) Phase shift

FIGURE 9: Derived features for $80 \mathrm{~g} / \mathrm{m}^{2}$ samples.

TABLE 2: Maximum, average, and variance of the relative errors for samples.

\begin{tabular}{|c|c|c|c|c|c|c|}
\hline & \multicolumn{3}{|c|}{$80 \mathrm{~g} / \mathrm{m}^{2}$} & \multicolumn{3}{|c|}{$160 \mathrm{~g} / \mathrm{m}^{2}$} \\
\hline & Peak amplitude & Delay time & Phase shift $(1.011 \mathrm{THz}) / \mathrm{rad}$ & Peak amplitude & Delay time & Phase shift $(1.392 \mathrm{THz}) / \mathrm{rad}$ \\
\hline Maximum (\%) & 35.12 & 13.0 & 3.61 & 30.22 & 6.27 & 1.74 \\
\hline Average (\%) & 12.19 & 3.59 & 1.39 & 6.30 & 3.05 & 0.58 \\
\hline Variance (\%) & 10.20 & 4.26 & 1.15 & 9.84 & 2.51 & 0.62 \\
\hline
\end{tabular}

Figure 11 shows the calculated relative errors of the three presented methods for $80 \mathrm{~g} / \mathrm{m}^{2}$ and $160 \mathrm{~g} / \mathrm{m}^{2}$ samples, respectively. Among the three presented methods, peakamplitude based methods have the largest relative errors in general. In the worst cases, the error is larger than $30 \%$, which does not meet the demands of quality control in modern papermaking industries. Generally, the relative errors of the delay time based method are around 6\%, whereas those of the phase-shift based method are around 2\%, particularly less than $5 \%$ in the worst case.

For further evaluation, the maximum, average, and variance of the relative errors were found and computed, as listed in Table 2. For the peak-amplitude based method, the maximum, average, and variance of the relative errors from $80 \mathrm{~g} / \mathrm{m}^{2}$ samples are $35.12 \%, 12.19 \%$, and $10.20 \%$, while the maximum, average, and variance are $13.0 \%, 3.59 \%$, and 


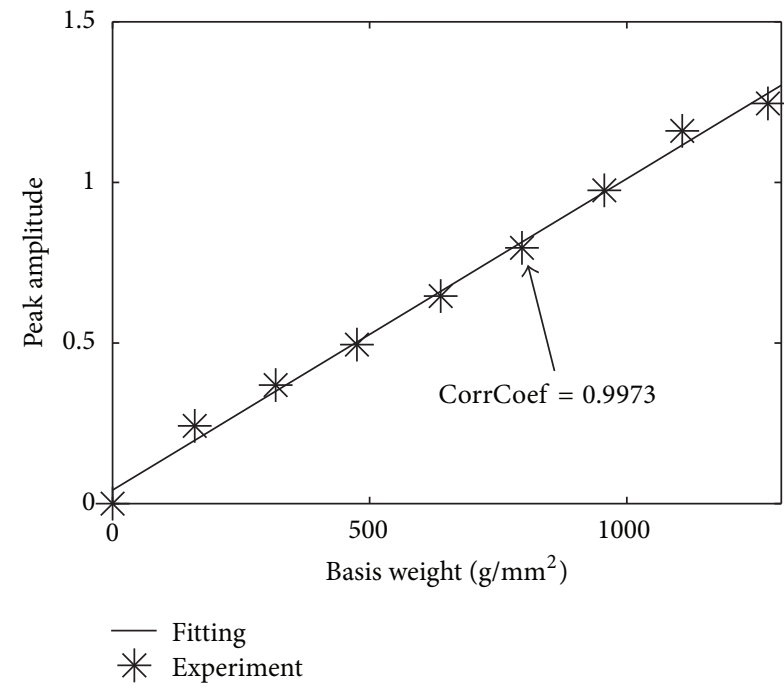

(a) Peak amplitude

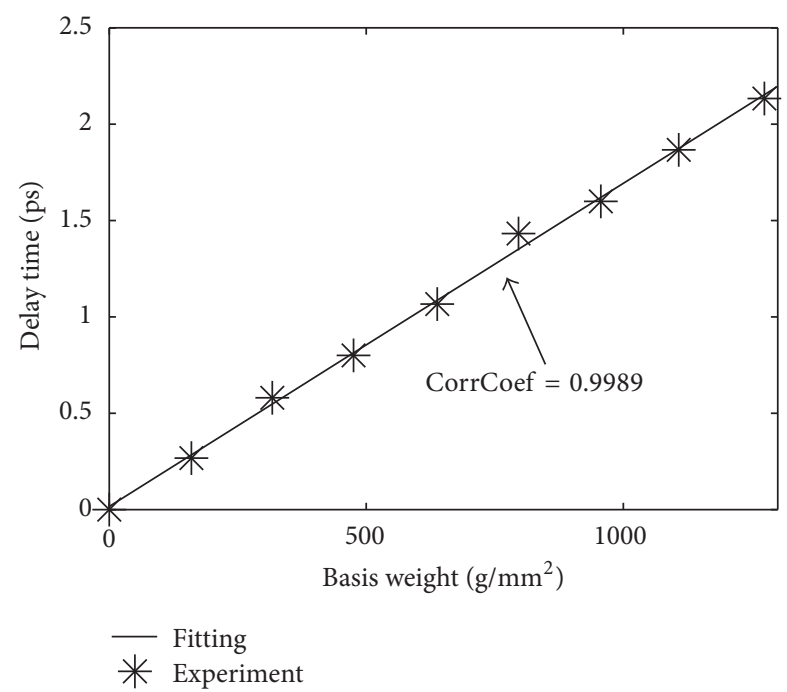

(b) Delay time

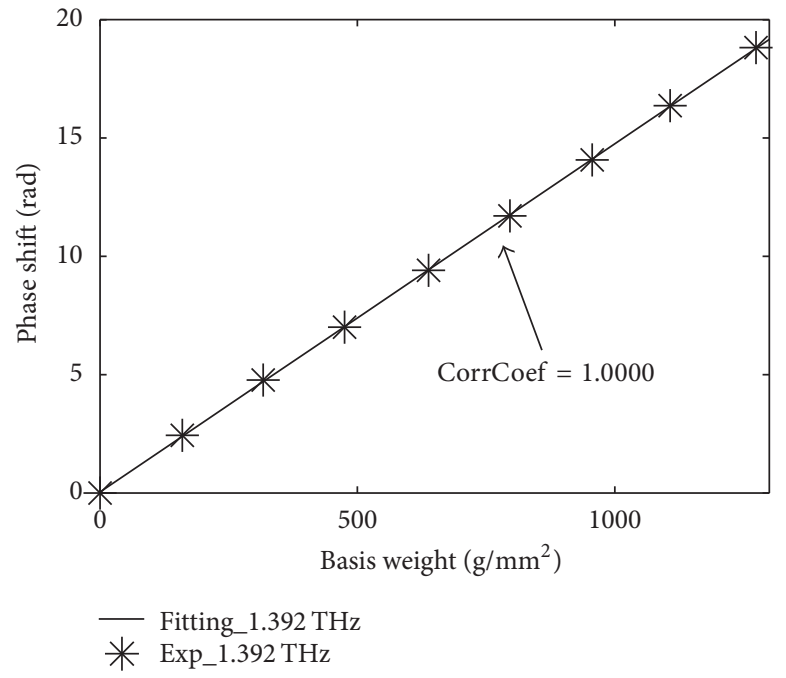

(c) Phase shift

FIGURE 10: Derived features for $160 \mathrm{~g} / \mathrm{m}^{2}$ samples.

$4.26 \%$ for the delay time based method. For the phaseshift based method, the maximum, average, and variance are $3.61 \%, 1.39 \%$, and $1.15 \%$ at $1.011 \mathrm{THz}$, respectively. In the same way, the maximum, average, and variance of the relative errors from $160 \mathrm{~g} / \mathrm{m}^{2}$ samples are $30.22 \%, 6.30 \%$, and $9.84 \%$ for the peak-amplitude based method, while the maximum, average, and variance are $6.27 \%, 3.05 \%$, and $2.51 \%$ for the delay time based method. For the phase-shift based method, the maximum, average, and variance are $1.74 \%, 0.58 \%$, and $0.62 \%$ at $1.392 \mathrm{THz}$, respectively. To sum up, the accuracy evaluation demonstrates that the phase-shift based method is significantly superior to the other two methods. Therefore, the use of phase-shift feature enhances the accuracy of paper basis weight measurement.

Basically, phase shift is dependent on refractive index and paper thickness, linearly related to paper basis weight. According to Fourier transform, delay time in the time domain is the combination of phase spectral change.
As a result, prediction error of phase shift at each frequency component shall contribute to that of delay time. This is the physics of phase shift superior to delay time.

4.4. Noise Robustness Evaluation. Harsh environments in industries will generate noises in the acquired $\mathrm{THz}$ pulses, and noise sensitivity has much effect on the uncertainty and reliability of measurements. Hence, we assessed noise robustness of the three aforementioned methods by artificially incorporating Gaussian noise into $\mathrm{THz}$ pulses. The incorporated Gaussian noises have $1.5 \mathrm{nA}, 2.5 \mathrm{nA}$, and $5.0 \mathrm{nA}$ standard deviations, respectively.

Figures 12 and 13 show the predicted errors with and without noises. For $80 \mathrm{~g} / \mathrm{m}^{2}$ samples, the maximum error of the peak-amplitude based method increases from $35.1 \%$ to $64.0 \%$, while that of the delay time based method increases from $13.0 \%$ to $48.7 \%$. For $160 \mathrm{~g} / \mathrm{m}^{2}$ samples, the maximum 


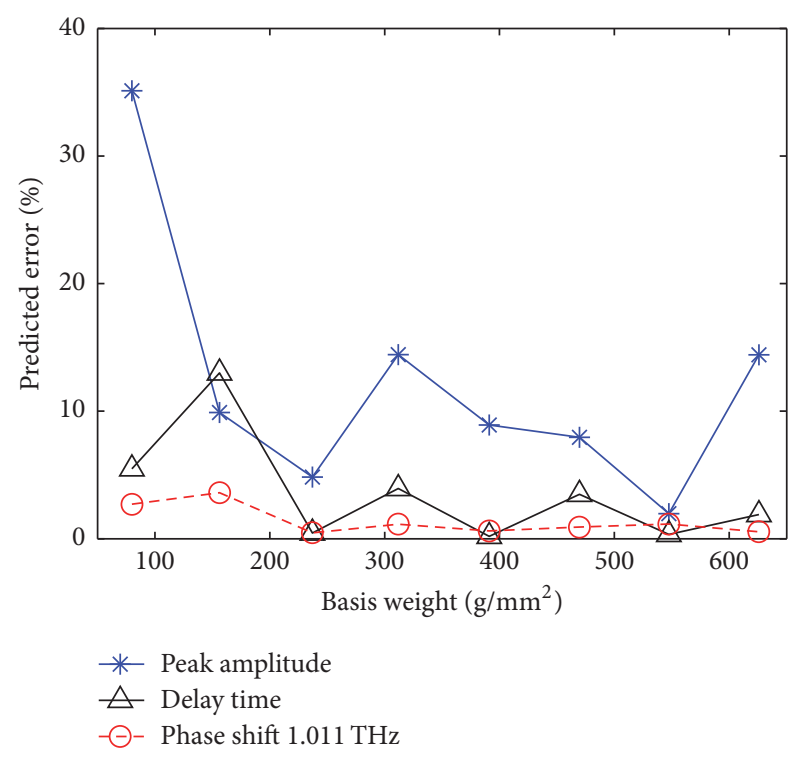

(a) $80 \mathrm{~g} / \mathrm{m}^{2}$ samples

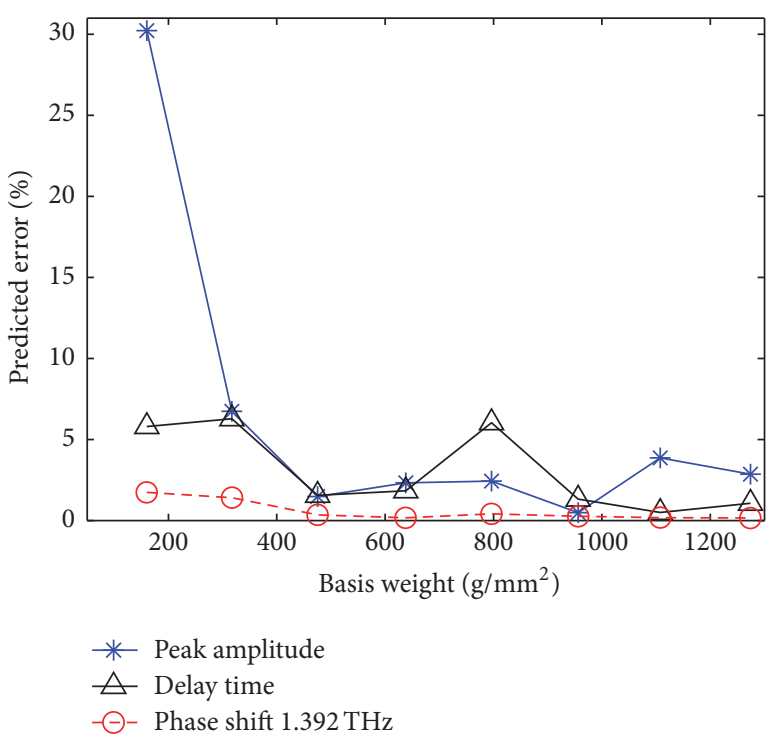

(b) $160 \mathrm{~g} / \mathrm{m}^{2}$ samples

FIGURE 11: Accuracy evaluation of the inferred paper basis weights.

error of the peak-amplitude based method increases from $30.2 \%$ to $95.7 \%$, while that of the delay time based method increases from $6.27 \%$ to $26.0 \%$. The maximum errors from both peak amplitude and delay time change dramatically with Gaussian noises. In contrast, the maximum errors of the phase-shift based method vary from $3.61 \%$ to $5.65 \%$ for $80 \mathrm{~g} / \mathrm{m}^{2}$ samples and from $1.74 \%$ to $7.09 \%$ for $160 \mathrm{~g} / \mathrm{m}^{2}$ samples, respectively.

The aforementioned analysis indicates that peak amplitude and delay time features are susceptible to noise contaminations. In contrary, the maximum predicted error of the phase-shift based method varies as well but slightly. In summary, phase shift is advantageous over peak amplitude and delay time in terms of noise robustness.

4.5. Discussions and Limitation. The sensitivity of the phaseshift based method increases with the increase of the frequency. The experimental setup Z-1 (Zomega Terahertz Corporation, New York, USA), which utilizes photoconductive antennas as generators and detectors [33], nominally ranges from $0.1 \sim 3.0 \mathrm{THz}$ in bandwidth. This hinders further improvement of the sensitivity. Recently, broadband or ultrabroadband THz-TDS instrument is commercially available and based on the generation and detection of $\mathrm{THz}$ pulses in femtosecond gas filaments or electrooptical generators and detectors [33]. Its advent extends the measurable frequency range from $3.0 \mathrm{THz}$ to $10 \mathrm{THz}$ or above, thus providing an advantage on the increase of the sensitivity for the phase-shift based method.

The inhomogeneity of the samples in thickness and paper basis weight was evaluated. For the inhomogeneity of the samples in thickness, we measured the thickness of 30 paper sheets at ten different points with a digital micrometer caliper. The ten readings show that the inhomogeneity of one paper sheet in thickness falls within $0.4 \mathrm{um}$. Paper basis weight is defined to the product of the mass density and thickness of paper sheets. To evaluate its inhomogeneity of nominal $80 \mathrm{~g} / \mathrm{m}^{2}$ samples, we also gauged the weight of 30 paper sheets with a $0.1 \mathrm{~g}$ fine balance. The average basis weight of the paper sheets is $79.90 \mathrm{~g} / \mathrm{m}^{2}$, and the mean deviation is $0.06 \mathrm{~g} / \mathrm{m}^{2}$. The results show that the inhomogeneity of the paper sheets in basis weight falls within $0.1 \mathrm{~g} / \mathrm{m}^{2}$ in general; thus the basis weight of the samples varies within $0.08 \%$. In conclusion, the used paper sheets could be regarded as homogeneous in all area of the samples in thickness and basis weight.

On the whole, high frequency of phase shift corresponds to high sensitivity. However, local fluctuations of the sensitivity are observed. To the authors' knowledge, the observed fluctuations are attributed to the variations of sample refractive index. It is reported that refractive index falls into the interval between 1.45 and 1.50 for paper sheet at the frequencies in the $\mathrm{THz}$ range $[30,34]$. The change of refractive index results from the dispersion of paper sheets. In addition, the multiple chemical substances and water content in paper sheets may contribute as well.

The selected frequency bands from $80 \mathrm{~g} / \mathrm{m}^{2}$ and $160 \mathrm{~g} / \mathrm{m}^{2}$ samples overlaps but partially. The main reasons behind this, to the authors' knowledge, may be the differences of chemical substances between $80 \mathrm{~g} / \mathrm{m}^{2}$ and $160 \mathrm{~g} / \mathrm{m}^{2}$ samples and inhomogeneity of the samples during the preprocessing of water vapor. It is well-known that paper sheets could be made of various types of raw materials like fibers, cotton, recycled paper, and used clothes. As a result, the chemical substances in paper sheets are somewhat different. The variation of the selected frequency band from different kinds of paper sheets demands recalibration on phase-shift gauge of paper basis weight, which is the limitation of the presented phase-shift based method. It is noted that the overlap between 


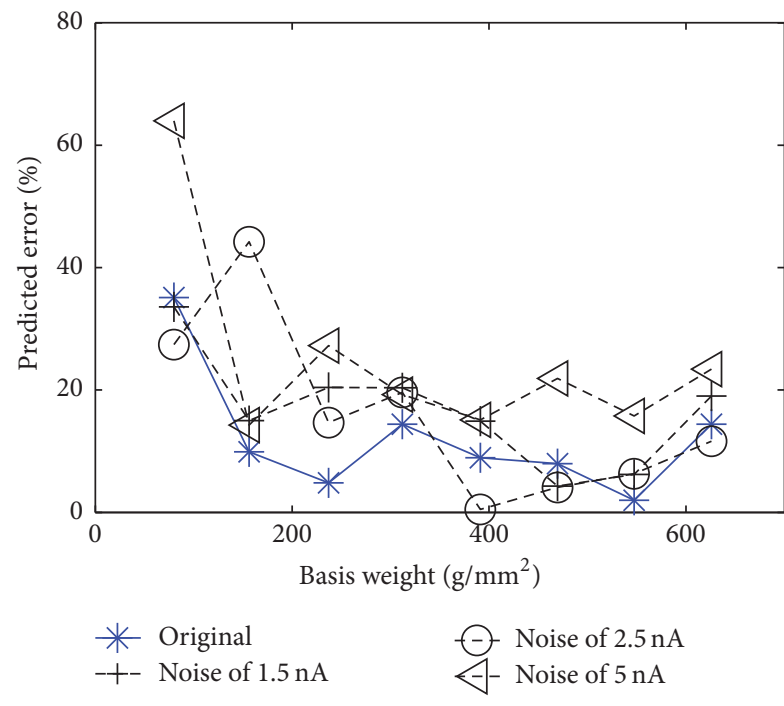

(a) Peak amplitude

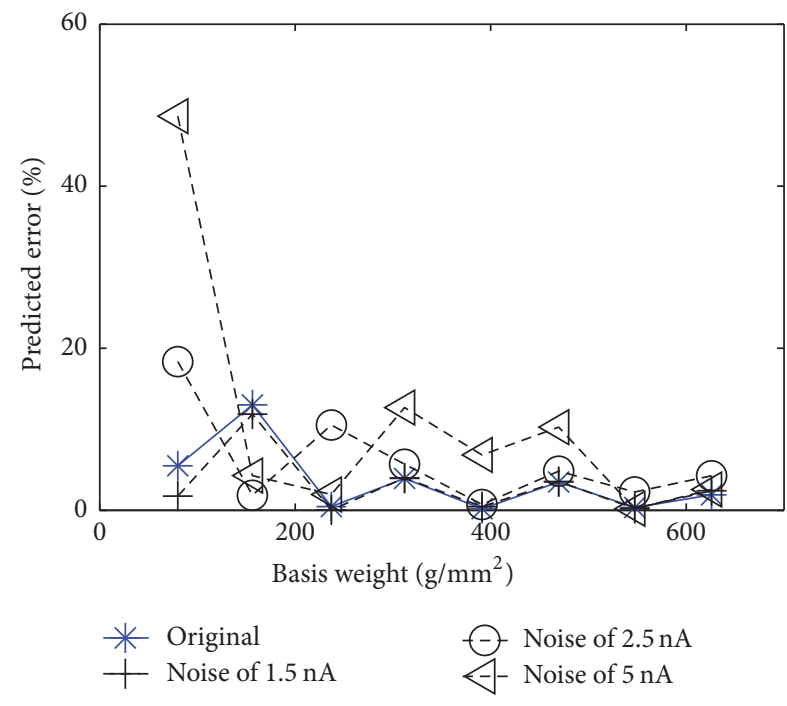

(b) Delay time

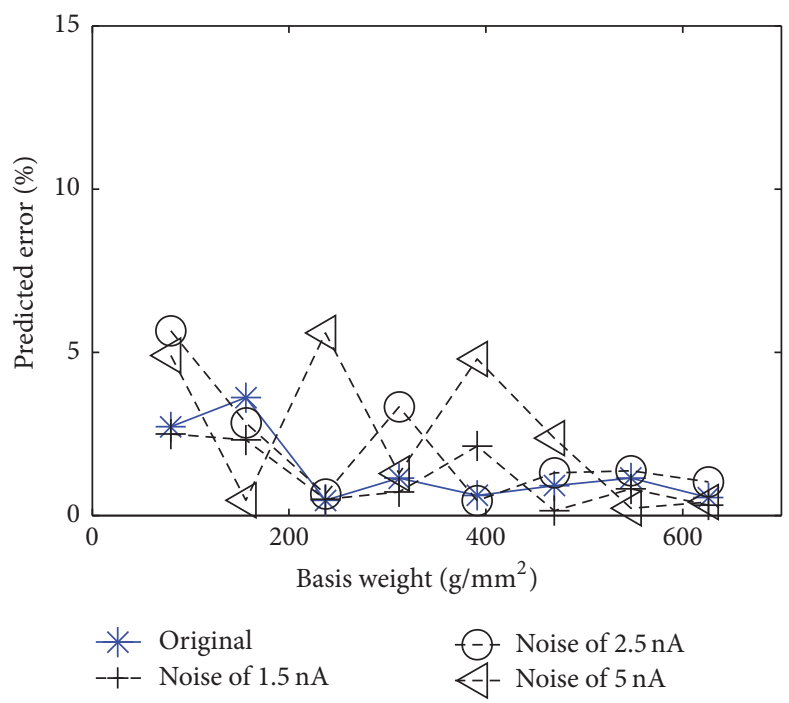

(c) Phase shift

FIGURE 12: Evaluation of noise robustness for $80 \mathrm{~g} / \mathrm{m}^{2}$ samples.

the selected frequency bands exists, which supports that the phase-shift based method is advantageous over the peakamplitude and delay time based methods.

The acquired $\mathrm{THz}$ pulses suffer from the variations generated by many sources of noises which are classified as random and systematic [29]. The contributions to random noises include laser intensity fluctuation, optical and electronic noise, delay line jitter, and so on, whereas systematic noises are introduced by registration error, mechanical drift, and so on. The methods to deal with the sources of noises are reported in many literatures. The variation in the amplitude of $\mathrm{THz}$ pulses is investigated to be a dominant contributor to the measurement error, since it may embrace the effects from the inhomogeneity of samples in nominal parameters. Theoretical formulations are also presented to quantify the amplitude-related variances.
There are hundreds of phase-shift signals available, as a matter of fact. Thus, we could adopt multiple satisfactory phase-shift features to infer paper basis weight simultaneously. In this way, the maximum errors could be reduced dramatically and random errors could be eliminated by means of information fusion techniques.

\section{Conclusion}

Phase shift of a $\mathrm{THz}$ pulse is proposed for determination of paper basis weight for accuracy improvement. Basically, phase shift, in the case of normal incidence, is a perfect feature to infer the basis weight of low-loss paper sheets attributed to the inherent linearity and noise insensitivity. Finally, the experimental results show that phase shift is feasible and advantageous over peak amplitude 


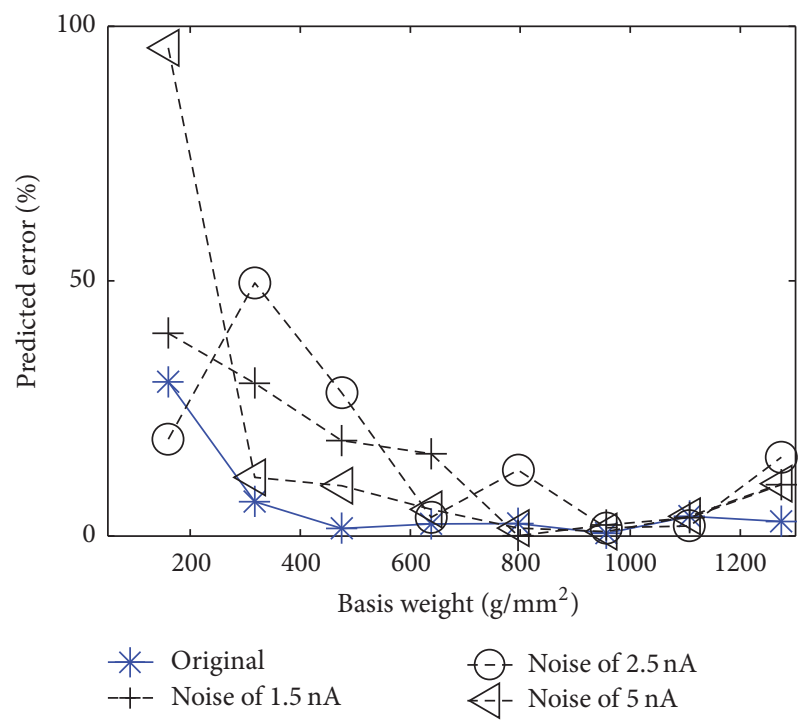

(a) Peak amplitude

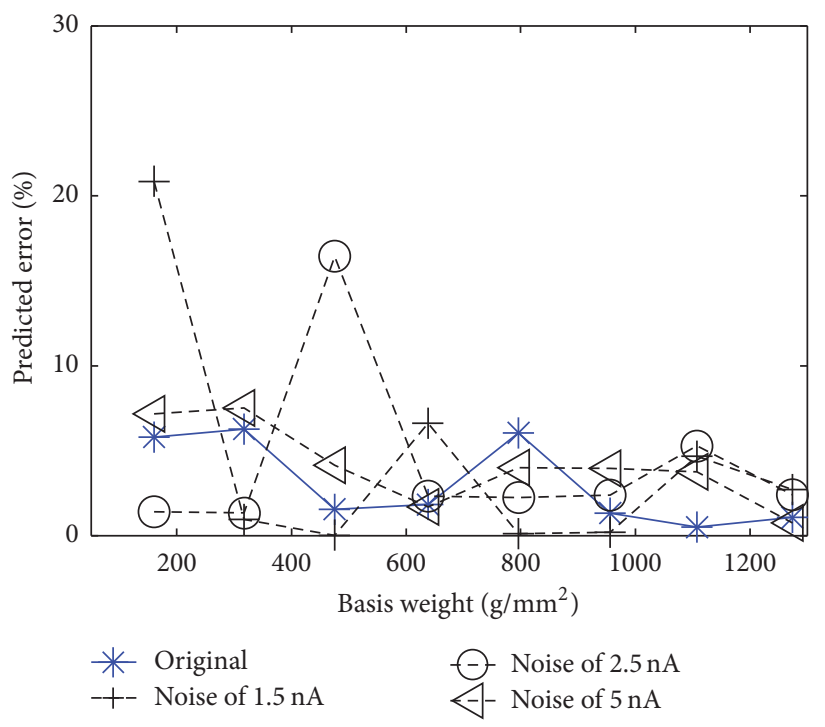

(b) Delay time

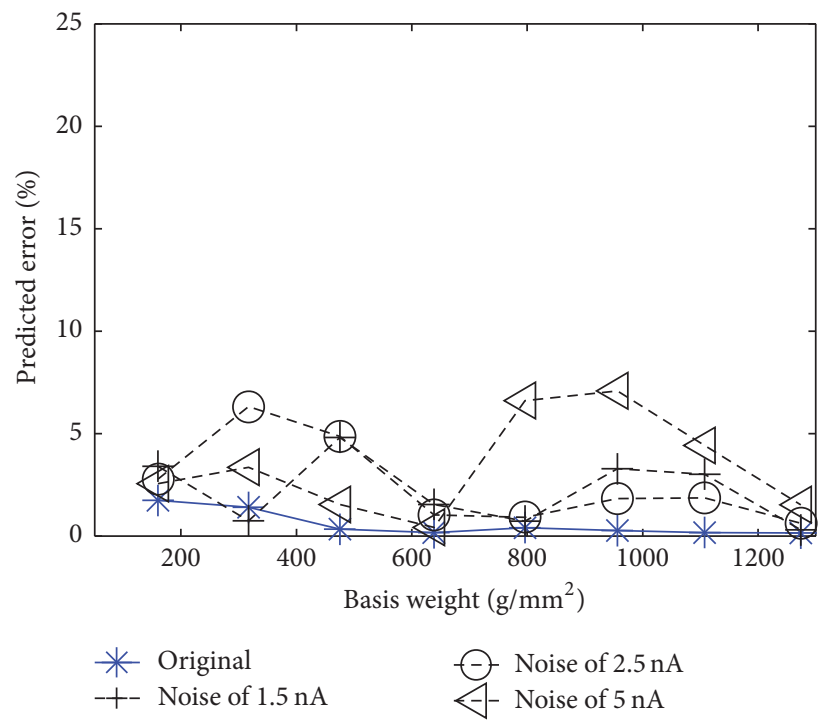

(c) Phase shift

FIGURE 13: Evaluation of noise robustness for $160 \mathrm{~g} / \mathrm{m}^{2}$ samples.

and delay time in terms of linearity, accuracy, and noise robustness.

The proposed method has significant advantages, such as noncontact, safety, simple signal processing, noise insensitivity, high resolution, and high speed. The experiments validated the capability of the proposed method for robust, noncontact, precise, and nonionizing measurement or online monitoring of paper basis weight or other plastic films for quality control at the manufacturing stage.

It is reported that paper is porous material, and the porosity of paper sheets could vary the complex refractive index. In theory, the proposed phase-shift based method applies to the measurement of paper porosity according to (5). In future, the research is scheduled to be carried out for feasibility verification.

\section{Conflicts of Interest}

The authors declare no conflicts of interest.

\section{Authors' Contributions}

Binghua Cao proposed the idea, designed the experiments, and cowrote the manuscript with Mengbao Fan; Mengbao Fan performed the experiments and analyzed the data; Guiyun Tian guided the research and was charged with the critical revisions of the manuscript.

\section{Acknowledgments}

This work is supported by Fundamental Research Foundation for the Central Universities under Grant 2013QNA47 and Six 
Talent Peaks Project in Jiangsu Province under Grant ZBZZ041. Dr. Binghua Cao and Dr. Mengbao Fan would like to acknowledge China University of Mining and Technology and Jiangsu Provincial Department of Education of China for sponsoring their visit to Newcastle University as academic visitors.

\section{References}

[1] X. Liu, M. Mwangi, X. Li, M. O’Brien, and G. M. Whitesides, "Paper-based piezoresistive MEMS sensors," Lab on a ChipMiniaturisation for Chemistry and Biology, vol. 11, no. 13, pp. 2189-2196, 2011.

[2] T.-K. Kang, "Tunable piezoresistive sensors based on pencil-onpaper," Applied Physics Letters, vol. 104, no. 7, Article ID 073117, 2014.

[3] D. D. Liana, B. Raguse, J. J. Gooding, and E. Chow, "Recent advances in paper-based sensors," Sensors, vol. 12, no. 9, pp. 11505-11526, 2012.

[4] R. Yowell, "On-line measurement and control of basis weight and thickness of rubber and plastic web products," in Proceedings of the IEEE Conference Record of 44th Annual Conference of Electrical Engineering Problems in the Rubber and Plastics Industries, pp. 40-46, Akron, Ohio, USA, April, 1992.

[5] C. Sharma and A. Jain, "Basis weight gain tuning using different types of conventional controllers," Bulletin Electrical Engineering Information, vol. 5, no. 1, pp. 62-71, 2016.

[6] Y. Shen, J. Q. Zhang, Y. Wang, and Z. Xinmin, "A new sensor for paper basis-weight and ash-content measurements," IEEE Transactions on Instrumentation and Measurement, vol. 46, no. 4, pp. 937-940, 1997.

[7] E. Belotserkovsky, J. A. Dahlquist, and P. Alto, "Coating weight measuring and control apparatus and method," United States Patent. 6074483, 2000.

[8] X. G. Wang, G. A. Dumont, and M. S. Davies, "Modelling and Identification of Basis Weight Variations in Paper Machines," IEEE Transactions on Control Systems Technology, vol. 1, no. 4, pp. 230-237, 1993.

[9] J. Stor-Pellinen, E. Hæggström, T. Karppinen, and M. Luukkala, "Air-coupled ultrasonic measurement of the change in roughness of paper during wetting," Measurement Science and Technology, vol. 12, no. 8, pp. 1336-1341, 2001.

[10] H. Ge, Y. Jiang, F. Lian, Y. Zhang, and S. Xia, "Characterization of wheat varieties using terahertz time-domain spectroscopy," Sensors, vol. 15, no. 6, pp. 12560-12572, 2015.

[11] M. Tonouchi, "Cutting-edge terahertz technology," Nature Photonics, vol. 1, no. 2, pp. 97-105, 2007.

[12] P. H. Siegel, "Terahertz technology," IEEE Transactions on Microwave Theory and Techniques, vol. 50, no. 3, pp. 910-928, 2002.

[13] W. Withayachumnankul, J. F. O’Hara, W. Cao, I. Al-Naib, and W. Zhang, "Limitation in thin-film sensing with transmissionmode terahertz time-domain spectroscopy," Optics Express, vol. 22, no. 1, pp. 972-986, 2014.

[14] C. L. K. Dandolo and P. U. Jepsen, "Wall painting investigation by means of non-invasive terahertz time-domain imaging (THz-TDI): inspection of subsurface structures buried in historical plasters," Journal of Infrared, Millimeter, and Terahertz Waves, vol. 37, no. 2, pp. 198-208, 2016.

[15] M. B. Fan, B. H. Cao, P. P. Yang, W. Li, and G. Y. Tian, "Elimination of liftoff effect using model-based method for eddy current characterization of a plate," NDT \& E International, vol. 74, pp. 66-71, 2015.

[16] L. Duvillaret, F. Garet, and J.-L. Coutaz, "Highly precise determination of optical constants and sample thickness in terahertz time-domain spectroscopy," Applied Optics, vol. 38, no. 2, pp. 409-415, 1999.

[17] P. Mousavi, F. Haran, D. Jez, F. Santosa, and J. S. Dodge, "Simultaneous composition and thickness measurement of paper using terahertz time-domain spectroscopy," Applied Optics, vol. 48, no. 33, pp. 6541-6546, 2009.

[18] M. Scheller and M. Koch, "Fast and accurate thickness determination of unknown materials using terahertz time domain spectroscopy," Journal of Infrared, Millimeter, and Terahertz Waves, vol. 30, no. 7, pp. 762-769, 2009.

[19] J. L. M. Van Mechelen, A. B. Kuzmenko, and H. Merbold, "Stratified dispersive model for material characterization using terahertz time-domain spectroscopy," Optics Letters, vol. 39, no. 13, pp. 3853-3856, 2014.

[20] C. D. Stoik, M. J. Bohn, and J. L. Blackshire, "Nondestructive evaluation of aircraft composites using reflective terahertz time domain spectroscopy," NDT \& E International, vol. 43, pp. 106115, 2010.

[21] E. V. Yakovlev, K. I. Zaytsev, I. N. Dolganova, and S. O. Yurchenko, "Non-destructive evaluation of polymer composite materials at the manufacturing stage using terahertz pulsed spectroscopy," IEEE Transactions on Terahertz Science and Technology, vol. 5, no. 5, pp. 810-816, 2015.

[22] A. Jagannathan, A. J. Gatesman, and R. H. Giles, "Characterization of roughness parameters of metallic surfaces using terahertz reflection spectra," Optics Letters, vol. 34, no. 13, pp. 1927-1929, 2009.

[23] A. Pohl, N. Deßmann, K. Dutzi, and H.-W. Hübers, "Identification of unknown substances by terahertz spectroscopy and multivariate data analysis," Journal of Infrared, Millimeter, and Terahertz Waves, vol. 37, no. 2, pp. 175-188, 2016.

[24] L. Zhang, Y. Zhang, C. Zhang, Y. Zhao, and X. Liu, “Terahertz multiwavelength phase imaging without $2 \pi$ ambiguity," Optics Letters, vol. 31, no. 24, pp. 3668-3670, 2006.

[25] Y. Wang, Z. Zhao, Z. Chen, L. Zhang, K. Kang, and J. Deng, "Continuous-wave terahertz phase imaging using a far-infrared laser interferometer," Applied Optics, vol. 50, no. 35, pp. 64526460, 2011.

[26] W. L. Chan, J. Deibel, and D. M. Mittleman, "Imaging with terahertz radiation," Reports on Progress in Physics, vol. 70, no. 8, pp. 1325-1379, 2007.

[27] N. V. Petrov, M. S. Kulya, A. N. Tsypkin, V. G. Bespalov, and A. Gorodetsky, "Application of terahertz pulse time-domain holography for phase imaging," IEEE Transactions on Terahertz Science and Technology, vol. 6, no. 3, pp. 464-472, 2016.

[28] B.-H. Cao, G.-X. Zhang, and Z.-K. Zhou, "New method for paper basis weight measurement based on terahertz time domain spectroscopy," Journal of Infrared and Millimeter Waves, vol. 28, no. 4, pp. 241-245, 2009.

[29] W. Withayachumnankul and M. Naftaly, "Fundamentals of measurement in terahertz time-domain spectroscopy," Journal of Infrared, Millimeter, and Terahertz Waves, vol. 35, no. 8, pp. 610-637, 2014.

[30] M. Reid and R. Fedosejevs, "Terahertz birefringence and attenuation properties of wood and paper," Applied Optics, vol. 45, no. 12, pp. 2766-2772, 2006. 
[31] Z. Xiaobo, Z. Jiewen, M. J. W. Povey, M. Holmes, and M. Hanpin, "Variables selection methods in near-infrared spectroscopy," Analytica Chimica Acta, vol. 667, no. 1-2, pp. 14-32, 2010.

[32] M. van Exter, C. Fattinger, and D. Grischkowsky, "Terahertz time-domain spectroscopy of water vapor," Optics Letters, vol. 14, no. 20, pp. 1128-1130, 1989.

[33] K. I. Zaytsev, A. A. Gavdush, V. E. Karasik et al., "Accuracy of sample material parameters reconstruction using terahertz pulsed spectroscopy," Journal of Applied Physics, vol. 115, no. 19, Article ID 193105, 2014.

[34] T. Hattori, H. Kumon, and H. Tamazumi, "Terahertz spectroscopic characterization of paper," in Proceedings of the 35th International Conference on Infrared, Millimeter, and Terahertz Waves (IRMMW-Thz '10), September 2010. 


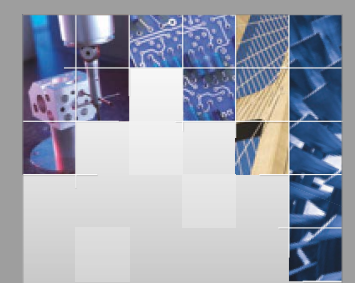

\section{Enfincering}
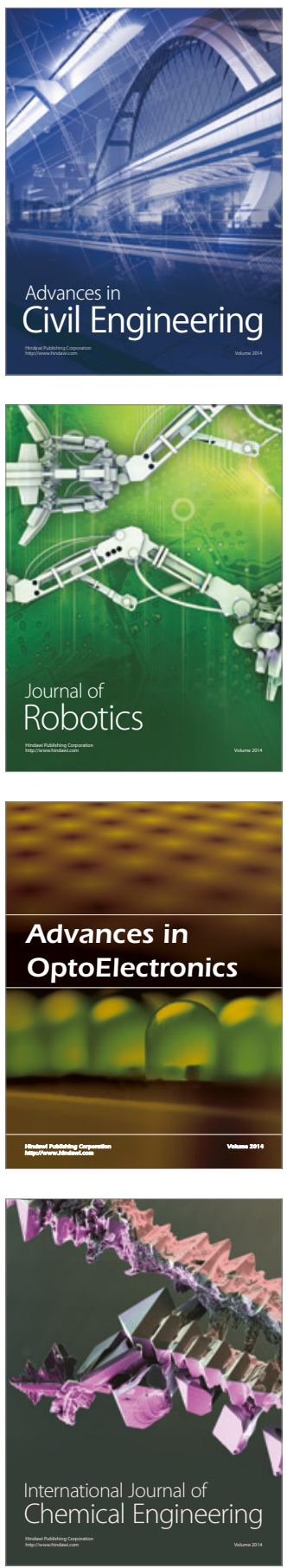

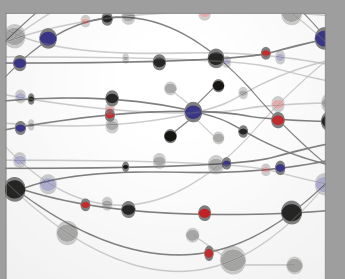

The Scientific World Journal

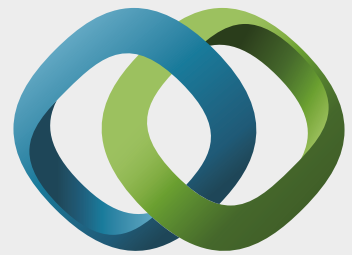

\section{Hindawi}

Submit your manuscripts at

https://www.hindawi.com
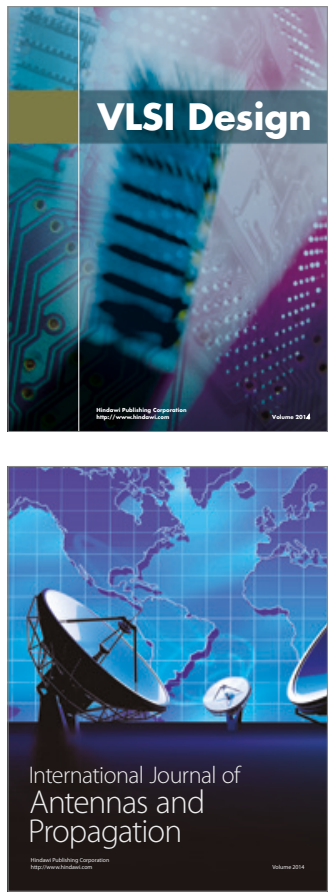

\section{Rotating}

Machinery
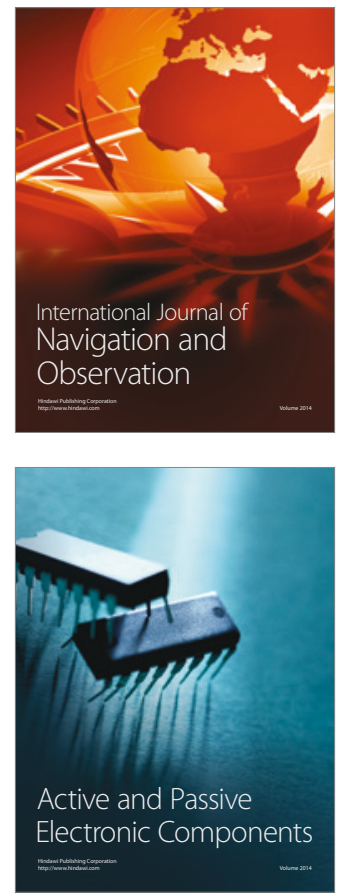
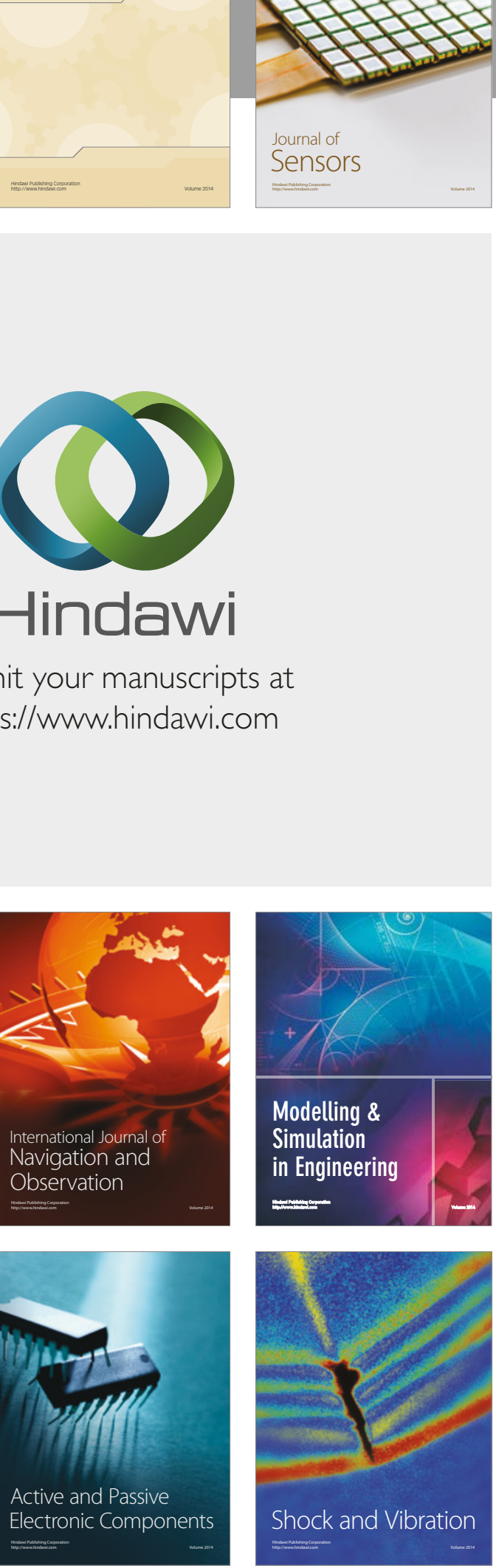
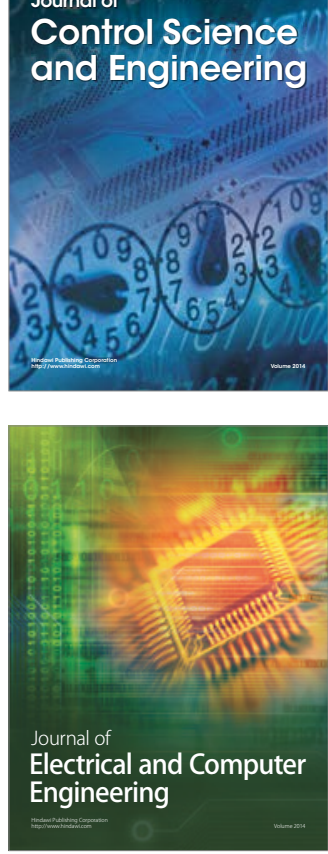

Distributed

Journal of

Control Science

and Engineering
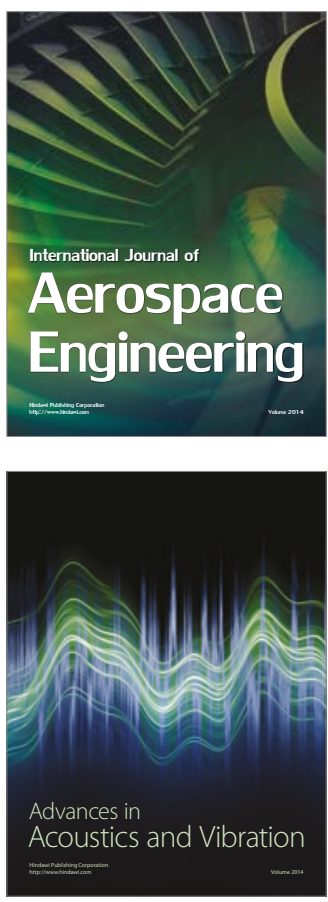

Sensor Networks 